\title{
Traumatic Incudostapedial Disarticulation: Anatomical Reconstruction Using Ionomeric Bone Cement
}

\author{
mohamed Ghonim ${ }^{1}$, Mohamad Salem², Yousef Shabana ${ }^{1}$, and Bassem Ashraf ${ }^{3}$ \\ ${ }^{1}$ Mansoura faculity of Medicine \\ ${ }^{2}$ Mansoura University \\ ${ }^{3}$ Mansoura Univerisity
}

May 7, 2020

Key Points

1. Traumatic ossicular lesions after head trauma are suspected in case of conductive hearing loss that does not resolve for more than six months with persistent air-bone gap (ABG) more than $30 \mathrm{~dB}$ with intact tympanic membrane.

2. The most common injury is incudostapedial joint disarticulation.

3. Tissue adhesive, cartilage, bone are utilized to manage the incudostapedial joint disarticulation while maintaining the normal anatomy.

4. The application of different bone cements has been increased in ear surgery.

5. Anatomical reconstruction of incudostapedial joint disarticulation using ionomeric bone cement is satisfactory cost-effective technique.

Introduction

Traumatic ossicular lesions after head trauma are suspected in case of conductive hearing loss that does not resolve for more than six months with persistent air-bone gap (ABG) more than $30 \mathrm{~dB}$ with intact tympanic membrane [1].

Different injuries can occur to the ossicular chain after head trauma. These injuries include incudostapedial joint disarticulation (ISD), displacement of incus and stapes lesions. It is generally known that the most frequent site to be injured is the icudostapedial joint. This is due to loose suspension of incus in-between both firmly attached stapes and malleus [2,3].

Several treatment modalities can be used for reconstruction of ISD. These include replacement of the IS joint or the incus completely using partial ossicular replacement prosthesis (PORP), in addition to incus interposition. Unfortunately, complications may occur after using these modalities as extrusion of prosthesis or migration of the graft, respectively. Alternatively, using prosthesis between intact incus and the footplate or the vestibule to bypass ISD and the stapes in cases with isolated ISD and or stapes lesions or both may solve this problems [3].

Tissue adhesive, cartilage, bone were utilized to manage the ISD while maintaining the normal anatomy. Also, the application of different bone cements has been increased in ear surgery which was introduced as an excellent alternative to currently accepted methods especially; for IS rebridging [4-6]. Cement plug technique was performed in revision stapedectomy cases in which long process of incus shows necrosis, the prosthesis was reinserted first onto remaining part of incus long process and then a plug of cement is used to fix it in place [7]. And using cement for anatomical repositioning of disarticulated incus in-between stapes and malleus during trans-mastoid supralabyrinthine facial decompression after temporal bone fractures [8]. 
This study was conducted to outline a surgical strategy, using ionomeric bone cement for anatomical reconstruction of post-traumatic isolated ISD with intact TM aiming to improve the stability and the functional outcome of ossiculoplasty.

Materials and Methods

Ethical Consideration:

This study was done in fulfillment of the requirements of the ethical committee of Otolaryngology Department and was approved by the Institutional Research Board of the Faculty of Medicine. Informed written consent was obtained from all patients.

This clinical study included 37 ears (37patients) operated upon for conductive hearing loss due to isolated ISD with intact TM following head trauma from 2011 to 2018. Anatomical reconstruction of ISD using ionomeric bone was performed.

Selection criteria: 1- Post-traumatic conductive HL due to isolated ISD with air bone gap, around 30. 2- Intact TM, 3- Lapse of 6 months or more after trauma, 4- No concurrent surgery for other pathology (tympanoplasty, facial nerve surgery).

Audiological assessment

Audiological assessment was done in consistence with the AAO-HNS Committee of Hearing and Equilibrium guidelines for treatment of conductive hearing loss. Preoperative and at least one year postoperative puretone audiometry was performed to all patients. Air conduction (AC) and bone conduction (BC) thresholds and $\mathrm{ABG}$ were documented at $0.5,1,2$, and $4 \mathrm{kHz}$. Postoperative ABG of [?]20 $\mathrm{dB}$ was considered as successful hearing result [9].

Surgical technique

All patients were operated by a senior surgeon. Trans-canal approach was used in all patients. The diagnosis of ossicular lesions depended mainly on the intraoperative finding. The middle ear and the ossicular chain status and mobility were examined microscopically and some cases, Otoendoscopes were used to exclude other ossicular lesions (Figures 1 and 2).

Reconstruction of ISD in 37 ears using ionomeric bone cement was done (Harvard Ionoglass Cem). After preparation of the cement, it was applied by a needle to bridge the gap and restore the integrity of IS joint anatomically. During application of cement, the footplate and facial nerve were covered with small pieces of gelfoam and then after the cement got hard, the gelfoam pieces were removed (Figures 3). The tympanic membrane was returned to its position. Then soaked gelfoam with antibiotic drops was used packed to the canal of external ear.

Statistical analysis was performed using a statistical software (SPSS, ver. 20, IBM, Chicago,IL USA). A paired Student's t-test was used to compare the means of the pre- and postoperative hearing results. Oneway ANOVA was used to compare means and standard deviations. Closure of air-bone gap within $20 \mathrm{~dB}$ was considered a successful result. $\mathrm{P}<0.05$ was considered significant

Results:

This study included 37 cases (37 ears). 35 cases (94.6\%) were males and $2(5.4 \%)$ were females. The mean age of cases was $21.13( \pm 10.5$; range, 12-45) years. The mean time for surgery after trauma was $4.9 \pm 5.2$ years (ranging from 8 months to 16.3 years). The commonest cause of trauma was traffic accidents in 30 cases $(81.1 \%)$. In the remaining 7 cases $(18.9 \%)$, the cause was fall from height and Blunt trauma to the head. The deafness was left sided in 31 ears (83.8\%) and right sided in 6 ears (16.2\%). 7 ears (18.9\%) had fracture line of posterior and post-superior meatal wall (Figure 1). Local anesthesia was used in $34(91.9 \%)$ ears. 
The mean preoperative $\mathrm{AC}$ and $\mathrm{BC}$ thresholds were $44.2+-12.8 \mathrm{~dB}$ and $11.6+-6.5 \mathrm{~dB}$. The mean postoperative $\mathrm{AC}$ and $\mathrm{BC}$ thresholds were $14.7+-11.4 \mathrm{~dB}$ and $11.3+-6.8 \mathrm{~dB}$. The mean pre- and postoperative ABGs were $32.5+-8.8$ and $3.3+-7.8 \mathrm{~dB}$ (table 1 ). The postoperative results were statistically significant for pure-tone average which improved by $30 \mathrm{~dB}(\mathrm{p}<0.001)$. The cochlear function remained stable postoperatively, there were no significant changes $(p=0.422)$.

There was significant improvement in the mean ABG from $32.4 \mathrm{~dB}$ preoperatively to $3.3 \mathrm{~dB}$ postoperatively $(\mathrm{p}<0.001)$. The ABG closure to within $10 \mathrm{~dB}$ was in 31 ears $(83.8 \%)$ and to within $20 \mathrm{~dB}$ in 33 ears $(89.2$ $\%$ ) and above $20 \mathrm{~dB}$ (mean $22 \mathrm{~dB}$ ) in 4 ears (10.8\%). There were no complications reported in all patients. There was no ABG more than $30 \mathrm{~dB}$ in any patient postoperatively.

The postoperative ABG closure was significantly better when surgical intervention was done before 2 years from the trauma (27 ears), it was closed to within $20 \mathrm{~dB}$ was all cases (100\%), while in 10 ears where surgical intervention done after 2 years only 6 ears $(60 \%)$ were achieved within $20 \mathrm{~dB}$ closure of ABG.

In 33 patients in whom $\mathrm{ABG}$ closure within $20 \mathrm{~dB}$ was achieved, the mean duration from onset of trauma to surgical intervention was $3.9+-4.2$ years. While in 4 patients who achieved more than $20 \mathrm{~dB}$ ABG closure, the duration from onset of trauma to surgical intervention was $16+-0.4$ years.

No complications were reported in any case regarding the bone cement application as perforation of tympanic membrane, granulations or otorrhea.

Discussion:

This study was conducted to assess the effectiveness of anatomical reconstruction of traumatic ISD using inomeric bone cement. In this study, the main cause of ISD was head trauma due to traffic accident which was reported by other studies [2,3]. In these cases, reconstruction of ISD using ionomeric bone cement represented an option.

In this study the overall postoperative results were statistically significant, regarding the pure-tone average, mean ABG and ABG closure. The cochlear function remained stable postoperatively, reflecting the safety and feasibility of this line of management. The ABG closure was statistically significantly improved when surgical intervention done early after trauma. A possible explanation can be that the delayed surgical intervention might lead to some sort of stapes fixation. There were no complications reported in all patients and there was no ABG more than $30 \mathrm{~dB}$ postoperatively.

Since earlier trials of Incudostapedial rebridging ossiculoplasty by reconstructing the missing part of the incus long process or ISD using liquid ionomeric bone cement, it had advantages of precise hearing results, cost effectiveness, and ease of application. In this study, the closure of ABG to within $20 \mathrm{~dB}$ was in 33 ears $(89.2 \%)$. Ozer et al, [6] found that in 9 out of 15 cases (60\%), successful hearing result was achieved (ABG to within $20 \mathrm{~dB}$ ). In a study, by Baglam et al. [5], on 136 patients with ISD, the postoperative ABG within $20 \mathrm{~dB}$ was attained in $81.6 \%$ of cases. There was significant improvement of the mean ABG and pure tone average postoperatively. The authors noted that the defect between the long process of incus and the head of stapes is important to consider in case selection; cases with defects less than one third of long process of incus are perfect candidates for reconstruction using cement. In another study, the long-term results of 233 cases who underwent ossicular reconstruction with Goldenberg hydroxyapatite prostheses (incus replacement, incus-stapes replacement, PORP and TORP) revealed successful hearing results in $50.6 \%$ of patients with an extrusion rate $5.29 \%$. The outcomes were best for incus replacement prosthesis (76\%) and the incus-stapes replacement prosthesis (85.7\%), with a significantly lower success rates for PORP (44.4\%) and the TORP (61.9\%). This study demonstrated lower success rates of PORP and TORP. [10]

\section{Conclusion:}

This study shows the effectiveness and safety of anatomical reconstruction of traumatic ISD using ionomeric bone cement when compared to conventional techniques.

Reference: 
1. Grant JR, Arganbright J, Friedland DR. Outcomes for Conservative Management of Traumatic Conductive Hearing Loss. Otol \& Neurotol 2008; 29:344-349.

2. Yetiser S, Hidir Y, Birkent H, Satar B, Durmaz A. A Traumatic ossicular dislocations: etiology and management. Am J of Otolaryngol Head Neck Med Surg 2008;29:31-36

3. Ghonim MR, Shabana YK, Ashraf B and Salem MB. Traumatic ossicular disruption with intact tympanic membrane: treatment modalities in 42 cases. Our experience in 42 patients. Clin Otolaryngol 2015;41:176-179.

4. Celik H, Felek SA, Islam A, Demirci M, Samim E, Oztuna D. The impact of fixated glass ionomer cement and springy cortical bone incudostapedial joint reconstruction on hearing results. Acta Otolaryngol 2009;129(12):1368-1373.

5. Baglam T, Karatas E, Durucu C, Kilic A, Ozer E, Mumbuc S, Kanlikama M. Incudostapedial Rebridging Ossiculoplasty with Bone Cement. Otolaryngol Head Neck Surg 2009;141:243-246.

6. Ozer E, Bayazit YA, Kanlikama M, Mumbuc S, Ozen Z. Incudostapedial Rebridging Ossiculoplasty with Bone Cement. Otol \& Neurotol 2002;23:643-646.

7. Ghonim MR, Shabana YK, Ashraf, B. Salem MB. Revision Stapedectomy with Necrosis of the Long Process of the Incus: Does the Degree of Necrosis Matter? A Retrospective Clinical Study. J Int Adv Otol 2017;13(1):28-31.

8. Ghonim MR, Shabana YK, Ashraf, B, Salem MB. Anatomical reposition of incus after transmastoid facial nerve decompression using bone cement: preliminary results in 17 patients. Clin Otolaryngol 2016;41(1):95-99.

9. Committee on Hearing and Equilibrium. Committee on Hearingand Equilibrium guidelines for the evaluation of results of treatment of conductive hearing loss. Otolaryngol Head Neck Surg 1995;113:186187.

10. Goldenberg RA, Driver M. Long-term results with hydroxylapatite middle ear implants. Otolaryngol Head Neck Surg 2000;122:635-642.

Table 1. Hearing results

\begin{tabular}{llll}
\hline Cases $=\mathbf{3 7}$ & Preoperative & Postoperative & Significance \\
\hline Air Conduction (dB) & $44+12.8$ & $14.7+11.4$ & $\mathrm{P}<0.001$ \\
Bone Conduction (dB) & $11.6+6.5$ & $11.3+6.8$ & $\mathrm{P}=0.422$ \\
Air-Bone Gap (dB) & $32.5+8.8$ & $3.3+7.8$ & $\mathrm{P}<0.001$ \\
\hline
\end{tabular}

Legends

Figure 1: it shows endoscopic view of the right middle ear with incudostapedial dislocation (black arrow), incus (I), stapes head (S) and fracture line (white arrow).

Figure 2: it shows microscopic view of the left middle ear with incudostapedial dislocation (black arrow), incus (I) and stapes head (S).

Figure 3: A\&B: it shows application of the cement by a needle to bridge the gap and restore the integrity of IS joint anatomically. C: it shows restored incudostapedial joint

\section{Hosted file}

table.docx available at https://authorea.com/users/319196/articles/448950-traumaticincudostapedial-disarticulation-anatomical-reconstruction-using-ionomeric-bone-cement 


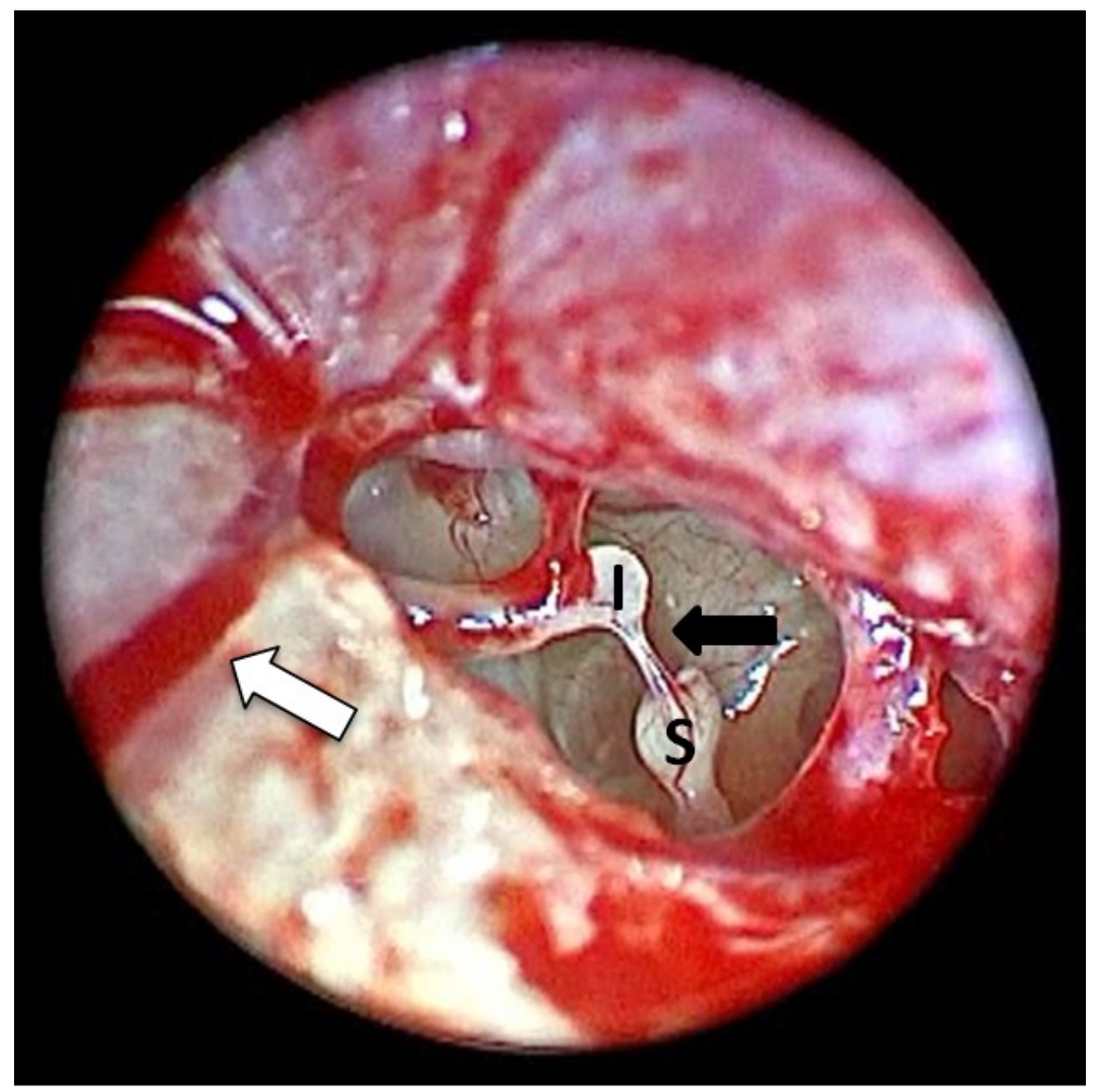




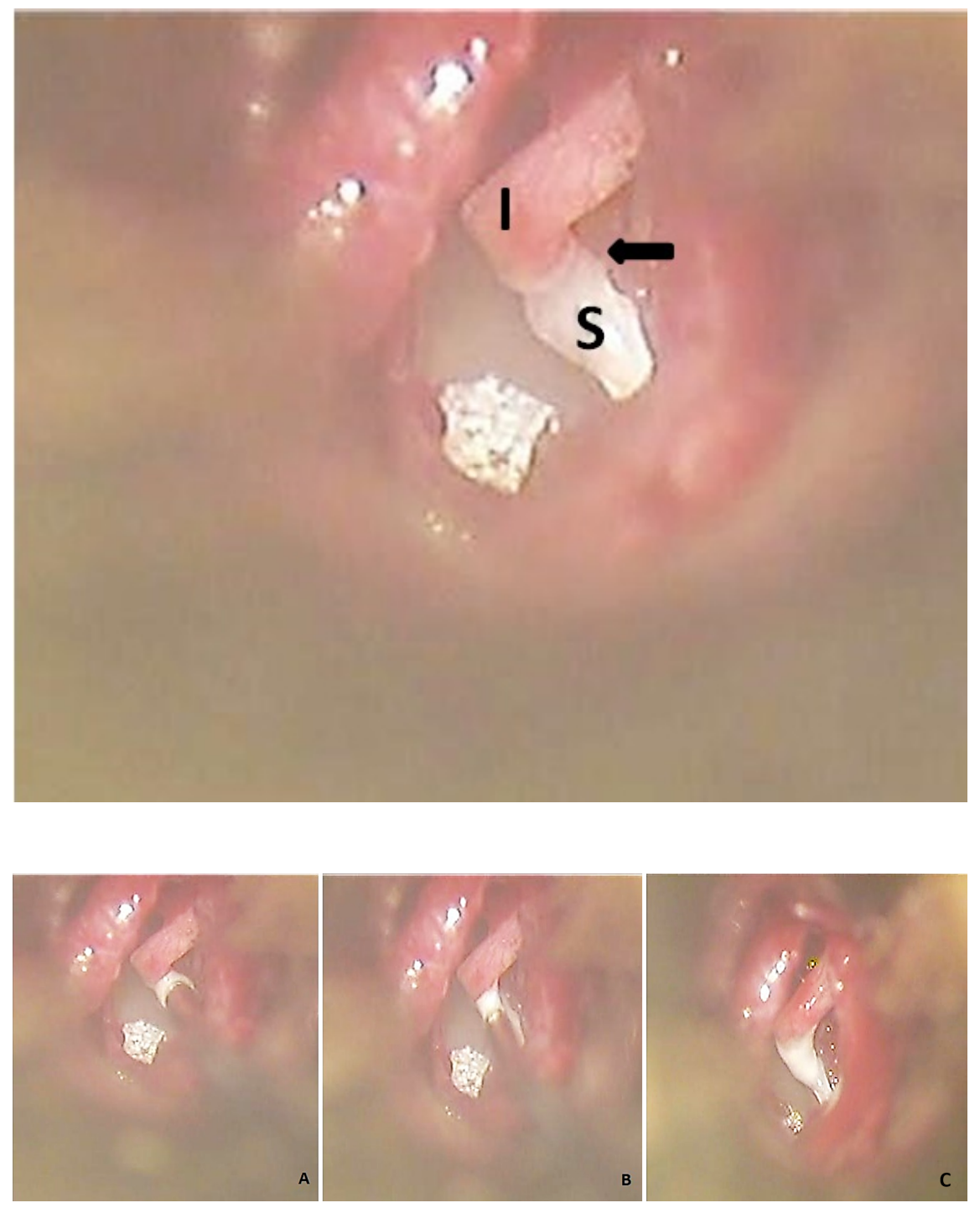

